\title{
Cálculo de la respuesta magneto-óptica de un ferrofluido
}

\author{
Ángel Sanz-Felipe, Juan Carlos Martín \\ Grupo de Tecnología Óptica Láser (TOL) \\ Instituto de Investigación en Ingeniería de Aragón (I3A) \\ Universidad de Zaragoza, Mariano Esquillor s/n, 50018, Zaragoza, Spain. \\ Tel. +34-976762707, e-mail: angel_sf@unizar.es
}

\section{Resumen}

Los ferrofluidos son materiales de gran interés para aplicaciones fotónicas. Su complejidad no permite predecir fácilmente sus propiedades ópticas. Proponemos un programa que simula la evolución temporal de las nanopartículas partiendo de sus parámetros relevantes. Con estas distribuciones simuladas se determinará la transmisión óptica para comparar con los resultados experimentales.

\section{Aplicaciones de ferrofluidos}

Los ferrofluidos son suspensiones coloidales de partículas magnéticas en un fluido. Sometidas a campo magnético, tienden a alinear sus momentos magnéticos y formar aglomerados, cadenas y grupos de cadenas por coalescencia lateral [1].

Estas sustancias presentan gran interés para aplicaciones fotónicas puesto que la aparición de aglomerados supone un cambio en la transmisión óptica del coloide con campos magnéticos pequeños. La capacidad de controlar sus propiedades ópticas los convierte en un elemento prometedor para el desarrollo de dispositivos de transmisión variable controlables a través de un campo magnético externo: reguladores de potencia, moduladores, conmutadores y sensores ópticos de campo magnético [2].

Sobre un volumen de ferrofluido, aplicamos campo magnético homogéneo en dirección paralela o perpendicular a la de la luz que incide en la muestra. $\mathrm{Su}$ respuesta magneto-óptica es altamente dependiente de diversos parámetros del ferrofluido como tamaño, magnetización de las partículas, concentración, viscosidad, propiedades del surfactante, etc. Por ello, resulta muy complejo predecir su comportamiento. En la Literatura se encuentran numerosos estudios experimentales que ofrecen diversos resultados. Los modelos teóricos y simulaciones son mucho más escasos y su capacidad de explicar resultados suele restringirse a unos pocos casos particulares o no alcanzan a una descripción completa. Así pues, no son fácilmente generalizables a otros ferrofluidos y quedan lejos de ofrecer una capacidad de predicción.

El objetivo de nuestro trabajo es encontrar un modelo completo que, considerando todas las interacciones dentro de un ferrofluido, permita describir mejor una sustancia tan compleja y ofrezca la capacidad de predecir su respuesta a partir de las características intrínsecas de la muestra en cuestión. Esto último se antoja fundamental de cara a seleccionar y optimizar un ferrofluido para sus aplicaciones.

Para ello, hemos desarrollado un programa que simula la evolución temporal de las partículas dentro del fluido como respuesta al campo magnético aplicado. En cada instante se puede determinar su transmisión óptica. En una etapa posterior, los resultados numéricos se compararán con los medidos en el laboratorio y con algún modelo teórico sencillo [3].

\section{Simulación de la evolución del ferrofluido}

El programa desarrollado permite determinar la evolución temporal de las nanopartículas en el fluido, es decir, posición, velocidad y orientación, partiendo de las características intrínsecas de la muestra. Para ello, el programa considera todas las interacciones físicas que sufren las partículas: fuerzas dipolo-dipolo entre partículas, fuerzas de repulsión por el surfactante estabilizador, interacción y giro por los campos magnéticos externo e interno, así como dispersión Browniana de traslación y rotación.

El programa sirve en primer lugar de base como análisis descriptivo de estos materiales: permite estudiar la influencia de cada parámetro en la formación o no de cadenas de partículas (Fig. 1), la velocidad del proceso, su longitud y distancia a que quedan las partículas, etc., dando muestra de la complejidad y sensibilidad de estos materiales a cualquier pequeña variación de sus características físicas. 


\section{Cálculo de la transmisión óptica}

El objetivo final de cara a aplicaciones fotónicas se debe completar con el cálculo de la transmisión óptica de la muestra simulada. Para ello, se toman distribuciones de partículas en distintos instantes a lo largo de la evolución previamente simulada. La transmisión óptica de cada una de estas distribuciones se calcula numéricamente mediante la herramienta BeamProp del programa RSoftCAD, que permite propagar una onda electromagnética por un medio con diferencias de índice de refracción, en este caso las partículas en el coloide.

Los cálculos de transmisión óptica del volumen de simulación se han comparado con los resultados experimentales obtenidos con un ferrofluido en particular cuyas principales características conocemos. Los primeros resultados corroboran la tendencia de los cambios de transmisión observada en el laboratorio en función de la orientación del campo, observándose un diferente scattering de luz (Fig. 2) que explica el aumento o disminución de transmisión. Por otro lado, se comprueba en las simulaciones que existe una dependencia en estos resultados con el grado de formación de cadenas y la separación final entre las partículas que las forman. Posteriormente, mediante la modificación de estas condiciones intrínsecas del ferrofluido, podremos ir ajustando las simulaciones a las medidas del laboratorio, completando así la descripción de la muestra utilizada. Estos ajustes servirán como puesta a punto de la simulación de cara a su utilización para predecir comportamientos de otros ferrofluidos y su optimización.

\section{Conclusiones}

Las simulaciones desarrolladas en cuanto a la evolución de las partículas de un ferrofluido y la transmisión resultante al propagar luz por él, arrojan unos resultados iniciales muy positivos contrastables con las medidas en el laboratorio y con algún modelo teórico sencillo. Esto implica que la simulación nos permitirá determinar algunos parámetros relevantes que aún desconocemos del ferrofluido analizado para su completa descripción. La puesta a punto completa de la simulación nos servirá para el estudio de ciertas dependencias de un ferrofluido y para su optimización de cara a aplicaciones fotónicas.

\section{REFERENCIAS}

[1]. IVEY, M., LIU, J., ZHU, Y. and CUTILLAS, S. Magnetic-field-induced structural transitions in a ferrofuid emulsion. Phys. Rev. E. 2000, 63, 011403.

[2]. PU, S., CHEN, X., CHEN, Y., XU, Y., LIAO, W., CHEN, L. and XIA, Y. Fiber-optic evanescent field modulator using a magnetic fluid as the cladding. $J$. Appl. Phys. 2006, 99, 093516.

[3]. SANZ-FELIPE, Á. and MARTÍN J. C. Analysis of the optical transmission of a ferrofluid by an electromagnetic mixture law. J. Phys. D: Appl. Phys. 2018, 51, 135001
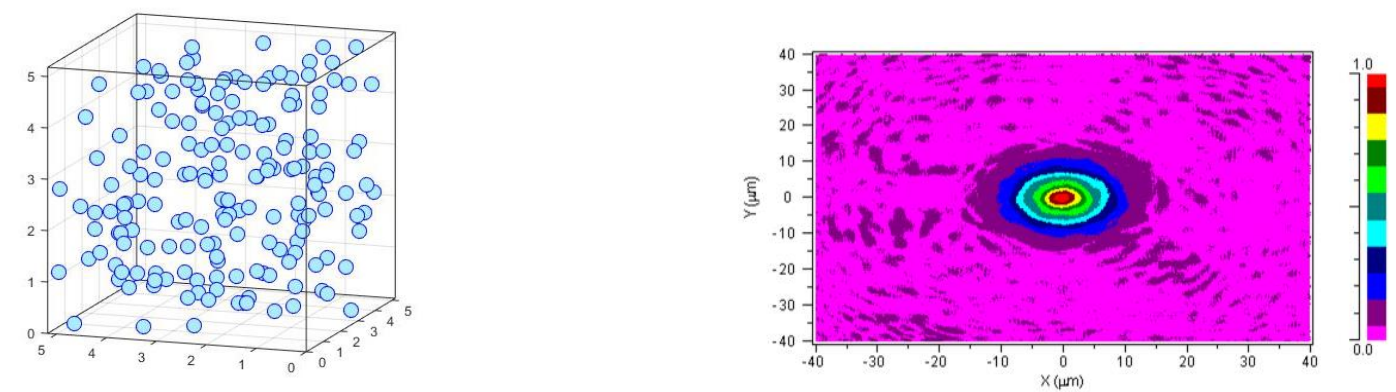

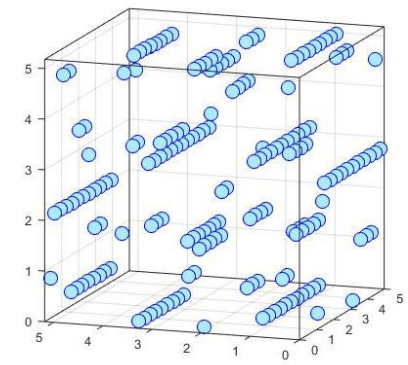

Fig. 1. Volumen de simulación inicial (arriba), y formación de cadenas al aplicar campo magnético (abajo).
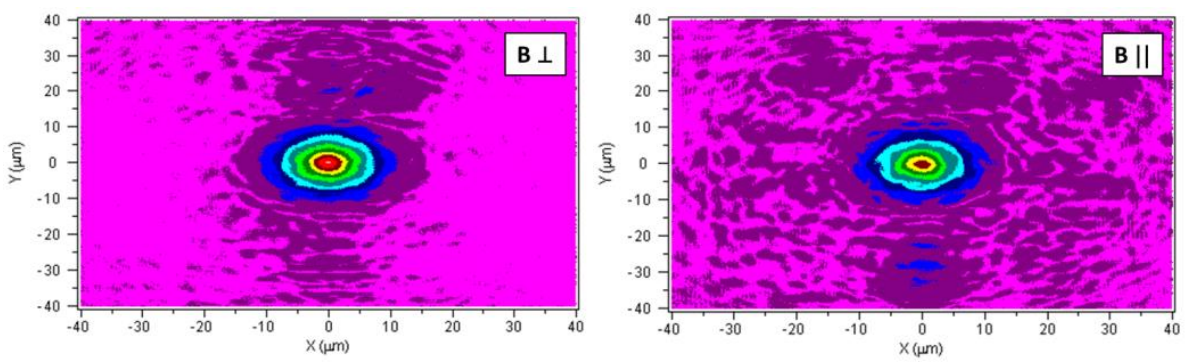

Fig. 2. Sección transversal de la transmisión de luz a partir del volumen de simulación inicial (arriba) y tras aplicar un campo magnético $\perp$ y $\|$ a la luz incidente (abajo). 